\title{
Kocuria Kristinae Meningitis and Cranial Nerve Palsies Secondary to Sphenoid Sinusitis: About a Case
}

\author{
Werdani Amina ${ }^{1}$, , Yahyaoui Omar ${ }^{1}$, Jammeli Nesrine ${ }^{1}$, Rassas Ahmed ${ }^{1}$, Boussofara Raoudha ${ }^{1}$, \\ Khedher Mohamed ${ }^{2}$, Mahjoub Bahri ${ }^{1}$
}

${ }^{1}$ Department of Pediatrics, Taher Sfar University Hospital, Mahdia, Tunisia

${ }^{2}$ Department of Microbiology, Taher Sfar University Hospital, Mahdia, Tunisia

Email address:

werdani_amina@yahoo.fr(W. Amina)

${ }^{*}$ Corresponding author

\section{To cite this article:}

Werdani Amina, Yahyaoui Omar, Jammeli Nesrine, Rassas Ahmed, Boussofara Raoudha, Khedher Mohamed, Mahjoub Bahri. Kocuria Kristinae Meningitis and Cranial Nerve Palsies Secondary to Sphenoid Sinusitis: About a Case. American Journal of Pediatrics.

Vol. 7, No. 3, 2021, pp. 159-162. doi: 10.11648/j.ajp.20210703.23

Received: July 25, 2021; Accepted: August 9, 2021; Published: August 23, 2021

\begin{abstract}
Kocuria spp, previously classified as the members of Micrococcacae family, was rarely reported as a human pathogen. Kocuria Kristinae could cause invasive infections of large variety of tissues in patients of any age. We report the first case of meningitis with sixth and third nerve palsies secondary to sphenoid sinusitis caused by Kocuria Kristinae in a previously healthy 13 year-old boy. In effect, his cerebrospinal fluid (CSF) showed a cloudy appearance of the CSF, white blood cells count was 600 cells $/ \mu 1$ (polymorphs $90 \%$ and $10 \%$ lymphocytes), red blood cells count was 200 cells $/ \mu 1$, protein level was elevated at $5.3 \mathrm{~g} / \mathrm{l}$ and glucose level was low at $0.1 \mathrm{mmol} / \mathrm{l}$. The direct examination showed positive-gram diplococci. The patient was initially treated with intravenous cefotaxim and vancomycin. The CSF culture was positive for gram-positive diplococci, which was identified as Kocuria kristinae. The meningitis was characterized by insidious evolution and persistent very low CSF glucose level. It was difficult to diagnose the sphenoid sinusitis because it is not accessible to direct clinical examination. It was diagnosed after the occurrence of a complication due to its anatomical location and proximity to the intracranial and orbital content. At day 5, the patient had remarkable resolution of symptoms. Complete recovery of cranial nerve palsy was noted at day 8. The aim of this case report is to present the first isolation of Kocuria Kristinae from cerebrospinal fluid sample and describe the clinical presentation and management outcomes.
\end{abstract}

Keywords: Kocuria Kristina, Meningitis, Sphenoid Sinusitis, Cranial Nerve Palsies

\section{Introduction}

Kocuria species are ubiquitous in the environment, commensal of the skin and mucosa [1]. Infectious pathologies caused by Kocuria species are uncommon and mostly present in immunocompromised individuals [2]. Meningitis caused by Kocuria is still limited in the literature. There are two documented cases of meningitis caused by Kocuria rosea [3, 4]. Here, we report a case of peadiatric bacterial meningitis with sixth and third nerve palsies secondary to sphenoid sinusitis. To the best of our knowledge, this is the first report of meningitis caused by Kocuria kristinae. The purpose of this case report is to describe the clinical presentation and management outcomes of this condition.

\section{Case Report}

A 13-year-old boy presented to our emergency department with headache, fever and asthenia of 8 days duration. Other symptoms were present including nausea, vomiting, photophobia and sleepiness. He had not no nasal obstruction nor rhinorrhea. The patient had no significant medical history, no ear discharge or any cranial surgery or trauma and no family history suggesting any significant illness.

The physical exam revealed poor general appearance and walking instability. He was febrile, with a temperature reaching $40^{\circ} \mathrm{C}$, heart rate of 93 beats per minute, polypnea with respiratory rate of 40 breaths per minute, blood pressure of $130 / 80 \mathrm{~mm} \mathrm{Hg}$ and transcutaneous oxygen saturation on 
room air of $96 \%$. His blood sugar test was $133 \mathrm{mg} / \mathrm{dl}$. Neurological examination revealed neck rigidity and Kernig's sign. He was able to speak coherently and his Glasgow coma scale was calculated at $15 / 15$. The oropharyngeal examination was normal, as was the rest of the physical examination. The funduscopic examination showed no papilledema. With this meningeal syndrome, blood cultures and a diagnostic lumbar puncture were performed prior to the administration of antibiotics. His cerebrospinal fluid (CSF) showed: a cloudy appearance of the CSF, white blood cells (WBC) count was 600 cells $/ \mu$ l (polymorphs $90 \%$ and $10 \%$ lymphocytes), red blood cells (RBC) count was 200 cells/ $\mu 1$, protein level was elevated at $5.3 \mathrm{~g} / \mathrm{l}$ and glucose level was low at $0.1 \mathrm{mmol} / \mathrm{l}$. The direct examination showed positive-gram diplococci.

Laboratory investigations revealed a total leukocyte count of $17490 / \mathrm{mm}^{3}$ (polymorphs $82,8 \%$ ), hemoglobin count of $11.3 \mathrm{~g} / \mathrm{dl}$. The $\mathrm{C}$ reactive protein was elevated up to $192 \mathrm{mg} / \mathrm{l}$, hyponatremia $(\mathrm{Na}+=131 \mathrm{mmol} / \mathrm{l})$ and hypokalemia $(\mathrm{k}+=2,57$ $\mathrm{mmol} / \mathrm{l})$. Renal and liver function tests were within normal limits.

The patient was initially treated with intravenous cefotaxim at the dose of $300 \mathrm{mg} / \mathrm{kg} /$ day (3 g every 6 hours), vancomycin $60 \mathrm{mg} / \mathrm{kg} /$ day (650 mg every 6 hours). The CSF culture was positive for gram-positive diplococci, which was identified as Kocuria kristinae.

The bacteria was sensitive to penicillin G, amoxicillin, cefotaxime, erythromycin, spiramycin, lincomycin, pristinamycin, ofloxacin, levofloxacin, teicoplanin and vancomycin but it had low-level bacterial resistance to streptomycin, kanamycin and gentamicin. Therefore, cefotaxim and vancomycin were continued.

At day 3, the patient had developped binocular diplopia, right ptosis (third right nerve palsy), an abduction deficit of the left eye (sixth left nerve palsy) (Figure 1).

Cerebral computed tomography (CT) demonstrated mucosal thickness and opacification of sphenoid sinuses. The brain was normal (Figure 2). Brain Magnetic Resonance Imaging (MRI) revealed acute sphenoiditis (Figure 3). So, the patient underwent endoscopic sphenoidotomy.

The fever persisted on day 4 of antibiotic treatment. So, a second lumbar puncture was performed that showed: WBC count was 620 cells/ $\mu$ l (polymorphs $80 \%$ and $20 \%$ lymphocytes), RBC count was 100 cells/ $\mu 1$, proteinorrachia at $1.84 \mathrm{~g} / \mathrm{l}$, hypoglycorrachia at $0.13 \mathrm{mmol} / \mathrm{l}$, direct examination and CSF culture were both negative. Chest x-ray, transthoracic echocardiography (TTE) and abdominal ultrasound were negative for infection.

At day 5, the patient had remarkable resolution of symptoms. Improvements were observed in a repeat CSF analysis. Both CSF and blood culture were negative. Complete recovery of cranial nerve palsy was noted at day 8 .

The patient was treated with IV cefotaxim and vancomycin for 2 weeks. He was discharged from the hospital within 17 days.

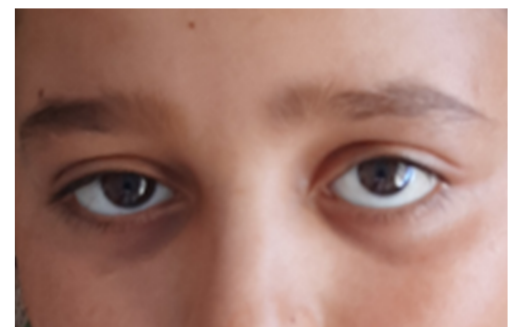

Figure 1. Photograph of the patient showing right ptosis (third right nerve palsy), an abduction deficit of the left eye (sixth left nerve palsy).

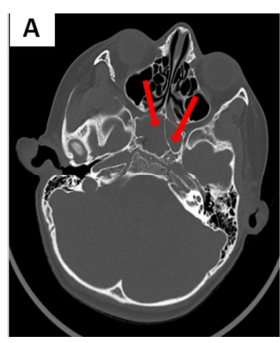

A: axial section
B: coronal section

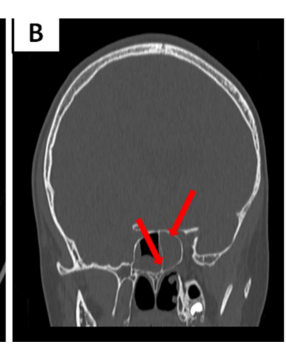

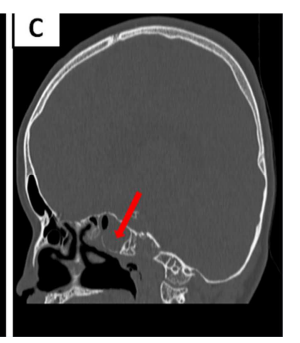

C: sagittal section
Figure 2. Brain computed tomography demonstrates fluid collection in the sphenoid sinuses.
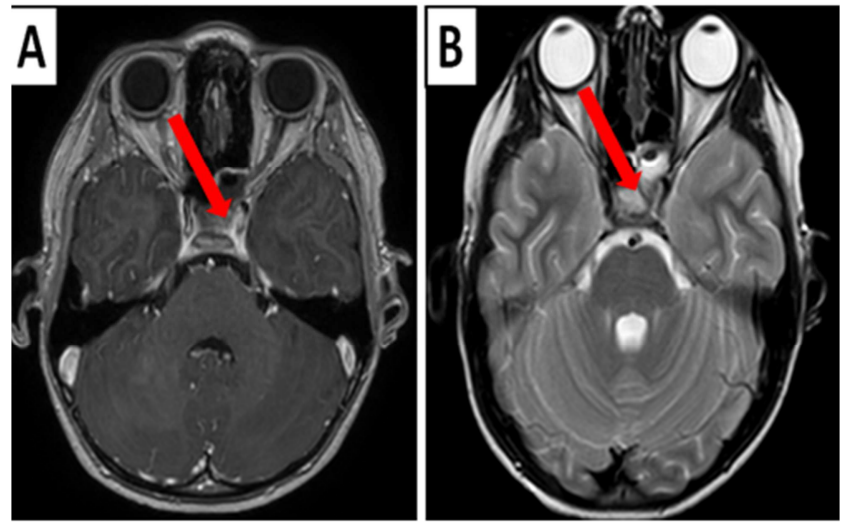

Figure 3. Brain Magnetic Resonance Imaging (MRI) showing sphenoid sinusitis (opacified sphenoid sinus and enhanced peripheral mucous membrane).

A: T1-weighted image axial section after gadolinium

B: T2-weighted image axial section

\section{Discussion}

Bacterial meningitis are associated with significant morbidity and risk of mortality. They are typically caused by virulent bacteria such as Streptococcus Pneumoniae, Neisseria Meningitidis. Streptococcus pneumoniae appears to be the pathogen most frequently associated with development of meningitis as a complication [5].

Kocuria Kristinae (or Micrococcus kristinae) is a Grampositive coccoid bacteria with tetrads often seen on smear, coagulase negative, oxidase positive and glucose fermenter positive [6]. It belongs to normal flora of skin and mucosa [6]. Infection is mostly associated with immunocompromised patients using intravenous catheter or peritoneal dialysis [2, 7]. Among the Kocuria species, K. kristinae, K. marina, K. 
rhizophila and K. varians have been observed to cause human infections [8]. It has been reported that Kocuria marina caused spontaneous bacterial peritonitis in a 2 year-old boy [9] and endocarditis in 10-year-old, immunocompetent girl [10]. Two cases of acute bacterial meningitis due to Kocuria rosea were reported $[3,4]$.

Only few cases infected with K. kristinae have been reported worldwide. The human infectious diseases caused by Kocuria kristinae are endocarditis [11], urinary tract infection [12], cholecystitis [13], peritonitis [7], bacteraemia [14], abdominal abscess [15], synovitis and periarticular bursitis [16], pneumonia [17], umbilical sepsis [18], bacteremic empyema [19] and interface keratitis [20]. We report the first case of meningitis caused by Kocuria kristinae, which was characterized by insidious evolution and persistent very low CSF glucose level.

Sphenoid sinusitis is a rare disorder of paranasal sinuses infection and most acute cases are related to Gram-positive cocci [21]. It appears to be difficult to diagnose because it is not accessible to direct clinical examination. There are many atypical presentations and may occur with complications due to its anatomical location and proximity to the intracranial and orbital content [22]. Sphenoid sinusitis may spread to the meninges or cavernous sinus via direct penetration [5]. It may present with isolated sixth nerve palsy [21-23] but also as with fulminant bacterial meningitis [5]. In a case series of 17 ocular cranial nerve palsies secondary to sphenoid sinusitis, the abducent nerve was the most common cranial nerve affected $(76 \%)$, followed by the oculomotor nerve (18\%) [24].

This is the first reported case of sphenoid sinusitis simultaneously complicated by both meningitis due to Kocuria Kristinae and ocular nerve palsy (right oculomotor and left abducens nerves).

Kocuria Kristinae is susceptible to amikacin, ampicillin, bacampecilin, cefazoline, abrekacin and debekacin, cefotaxime, ceforixim, clarythromycin, clindamycin, dalbavancin, doripenem, fusidic acid, gentamicin, imipenem, isepamicin, lincomycin, meropenem, meropenem/veborbactam, netilmicin, penicillin $\mathrm{G}$, rifampin, rifaximin, teicoplanin and vancomycin [6].

Initial treatment of Kocuria Kristinae infections should involve parenteral vancomycin in combination with some other antibiotic to which this bacteria is susceptible [25].

\section{Conclusion}

Sphenoid sinusitis is uncommon paranasal sinuses infection. Intracranial complications of sinusitis, including meningitis, ocular nerve palsy are severe and associated with significant morbidity. In immunocompromised but also immunocompetent patients, unusual organisms such as Kocuria Kristinae may cause significant infection. Imaging of the brain should be performed in all cases with insidious fever and headache, to rule out causes such as sphenoid sinusitis.

\section{References}

[1] Stackebrandt E, Koch C, Gvozdiak O, Schumann P. Taxonomic dissection of the genus Micrococcus: Kocuria gen. nov., Nesterenkonia gen. nov., Kytococcus gen. nov., Dermacoccus gen. nov., and Micrococcus Cohn 1872 gen. emend. Int J Syst Bacteriol. oct 1995; 45 (4): 682-92.

[2] Dunn R, Bares S, David MZ. Central venous catheter-related bacteremia caused by Kocuria kristinae: case report and review of the literature. Ann Clin Microbiol Antimicrob. 24 août 2011; 10: 31 .

[3] Paul M, Gupta R, Khush S, Thakur R. Kocuria rosea: An emerging pathogen in acute bacterial meningitis- Case report.: 4.

[4] Sipahi OR, Mermer S, Aydemir S, Ozgiray E, Cilli F, Oner K. Kocuria rosea meningitis. Surg Infect (Larchmt). oct 2014; 15 (5): 659 .

[5] Saitoh A, Beall B, Nizet V. Fulminant bacterial meningitis complicating sphenoid sinusitis. Pediatric Emergency Care. déc 2003; 19 (6): 415-7.

[6] Inc GI, Berger DS. GIDEON Guide to Medically Important Bacteria. GIDEON Informatics Inc; 2020. 2080 p.

[7] Cheung CY, Cheng NHY, Chau KF, Li CS. An Unusual Organism for CAPD-Related Peritonitis: Kocuria kristinae. Perit Dial Int. 1 janv 2011; 31 (1): 107-8.

[8] Chakraborty DB, Banerjee DD. COMBATING KOCURIA: A GAME OF SHADOWS. International Journal of Scientific Research [Internet]. 21 janv 2020 [cité 30 août 2020]; 8 (12). Disponible sur: http://www.worldwidejournals.org/index.php/ijsr/article/view/ 945.

[9] Brändle G, L'Huillier AG, Wagner N, Gervaix A, Wildhaber BE, Lacroix L. First report of Kocuria marina spontaneous peritonitis in a child. BMC Infect Dis. 30 déc 2014; 14: 719.

[10] Moreira JS, Riccetto AGL, Silva MTN da, Vilela MM dos S, Moreira JS, Riccetto AGL, et al. Endocarditis by Kocuria rosea in an immunocompetent child. Brazilian Journal of Infectious Diseases. févr 2015; 19 (1): 82-4.

[11] Lai CC, Wang JY, Lin SH, Tan CK, Wang CY, Liao CH, et al. Catheter-related bacteraemia and infective endocarditis caused by Kocuria species. Clinical Microbiology and Infection. 1 févr 2011; 17 (2): 190-2.

[12] Tewari R, Dudeja M, Das AK, Nandy S. Kocuria Kristinae in Catheter Associated Urinary Tract Infection: A Case Report. J Clin Diagn Res. août 2013; 7 (8): 1692-3.

[13] Ma ES, Wong CL, Lai KT, Chan EC, Yam W, Chan AC. Kocuria kristinae infection associated with acute cholecystitis. BMC Infectious Diseases. 19 juill 2005; 5 (1): 60.

[14] Basaglia G, Carretto E, Barbarini D, Moras L, Scalone S, Marone P, et al. Catheter-Related Bacteremia Due to Kocuria kristinae in a Patient with Ovarian Cancer. Journal of Clinical Microbiology. 1 janv 2002; 40 (1): 311-3.

[15] Kolikonda MK, Jayakumar P, Sriramula S, Lippmann S. Kocuria kristinae infection during adalimumab treatment. Postgraduate Medicine. 17 févr 2017; 129 (2): 296-8. 
[16] Foli M, Rosi N. Synovitis And Periarticular Bursitis Of The Coxofemoral Joint Caused By Kocuria Kristinae: A Case Report. ACTA FACULTATIS MEDICAE NAISSENSIS. 2010; 27 (1): 6.

[17] Bernshteyn M, Kumar PA, Joshi S. Kocuria kristinae pneumonia and bacteremia. Baylor University Medical Center Proceedings. 23 juill 2020; 0 (0): 1-2.

[18] Sivaraman U, Subramanian P, Ramakrishnan K, Seetha KS. Umbilical Sepsis Caused by Multidrug Resistant Strain of Kocuria kristinae in a New Born: A Case Report. J Clin Diagn Res. avr 2016; 10 (4): DD01-2.

[19] Kim KY, Cho JH, Yu CM, Lee KJ, Lee JM, Koh S, et al. A Case of Community-acquired Bacteremic Empyema Caused by Kocuria kristinae. Infect Chemother. juin 2018; 50 (2): $144-8$.

[20] Kate A, Joseph J, Bagga B. Kocuria kristinae interface keratitis following deep anterior lamellar keratoplasty. Indian J Ophthalmol. juill 2020; 68 (7): 1463-6.

[21] Del Brutto OH, Caputi R. Isolated abducens palsy due to acute sphenoid sinusitis. International journal of infectious diseases: IJID: official publication of the International Society for Infectious Diseases. 2018; 72: 6-7.

[22] Gupta R, Shukla R, Mishra A, Parihar A. Isolated acute sphenoid sinusitis presenting with hemicranial headache and ipsilateral abducens nerve palsy. BMJ case reports. 8 juin $2015 ; 2015$.

[23] Ada M, Kaytaz A, Tuskan K, Güvenç MG, Selçuk H. Isolated sphenoid sinusitis presenting with unilateral VIth nerve palsy. International Journal of Pediatric Otorhinolaryngology. avr 2004; 68 (4): 507-10.

[24] El Mograbi A, Soudry E. Ocular cranial nerve palsies secondary to sphenoid sinusitis. World Journal of Otorhinolaryngology - Head and Neck Surgery. mars 2017; 3 (1): 49-53.

[25] Zarić RSŽ, Pejčić AV, Janković SM, Kostić MJ, Milosavljević MN, Milosavljević MJ, et al. Antimicrobial treatment of Kocuria kristinae invasive infections: Systematic review. Journal of Chemotherapy. 3 avr 2019; 31 (3): 109-19. 